\title{
Toroidal Plasmonic Eigenmodes in Oligomer Nanocavities for the Visible Detected by EFTEM and 3D-FDTD Simulations
}

\author{
Burcu Ögüt ${ }^{1}$, Nahid Talebi ${ }^{1}$, Ralf Vogelgesang ${ }^{2}$, Wilfried Sigle ${ }^{1}$, Peter A. van Aken ${ }^{1}$ \\ 1. Max-Planck-Institute for Intelligent Systems, 70569 Stuttgart, Germany. \\ 2. Carl von Ossietzky University of Oldenburg, 26129 Oldenburg, Germany.
}

Miniaturization has become one of the pillars of modern technology due to the trend of the electronic devices' diminishing down to the nanoscale since the beginning of the last decade. Using light for information technology in such devices is impeded by the diffraction limit of light. Converting light into surface plasmons could be a way to circumvent this limit because plasmons have much smaller wavelengths. Therefore plasmonics has become an astonishingly expanding field of technology, which offers numerous possibilities for applications beyond the diffraction limit of light.

Many plasmonic applications are based on electric-dipole and higher-order multipole resonances, as well as magnetic resonances. Here, by energy-filtering transmission electron microscopy (EFTEM) supported with 3D finite-difference time domain (3D-FDTD) simulations we detect the signatures of toroidal resonances in oligomer nanocavities at optical wavelengths, which could not be captured by conventional optical microscopy techniques due to their nearly radiation-free nature. Figure 1 displays a schematic illustration of a toroidal moment. A toroidal moment is composed of combination of electrical and magnetic field loops revolving in radial and azimuthal directions, respectively [1].

Figure 2 illustrates the power of an electron beam to excite various plasmonic eigenmodes, normally requiring different optical excitation setups, all at once. Figure 2a, 2b, and $2 \mathrm{c}$ display the acquired EFTEM images, corresponding peak maps, and the results of 3D-FDTD simulations, respectively. Modes II and III reveal the modes having magnetic and electrical dipolar character, accordingly, whereas modes I and IV correspond to toroidal modes. Even if the central hole is removed, evidence of toroidal behaviour persists, which would be totally impossible to capture with an optical microscopy technique [1].

We believe that toroidal moments have a big potential for novel innovations in the future, such as Purcell effect engineering [2] and data storage and processing [3, 4].

\section{References}

[1] B Ögüt et al, Nano Letters 12 (2012), 5239.

[2] KJ Vahala, Nature 424 (2003), 839.

[3] KS Choi et al, Nature 452 (2008), 67.

[4] The research leading to these results has received funding from the European Union Seventh Framework Programme [FP7/2007- 2013] under grant agreement n³12483 (ESTEEM2). 


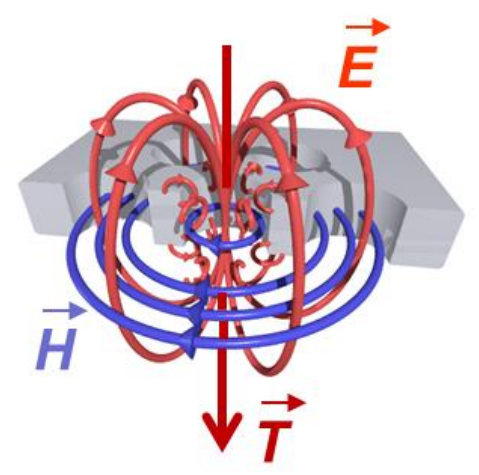

Figure 1. Illustration of a toroidal moment in a plasmonic nanocavity system formed by electric (red) and magnetic field (blue) whirling in radial and azimuthal direction, respectively.

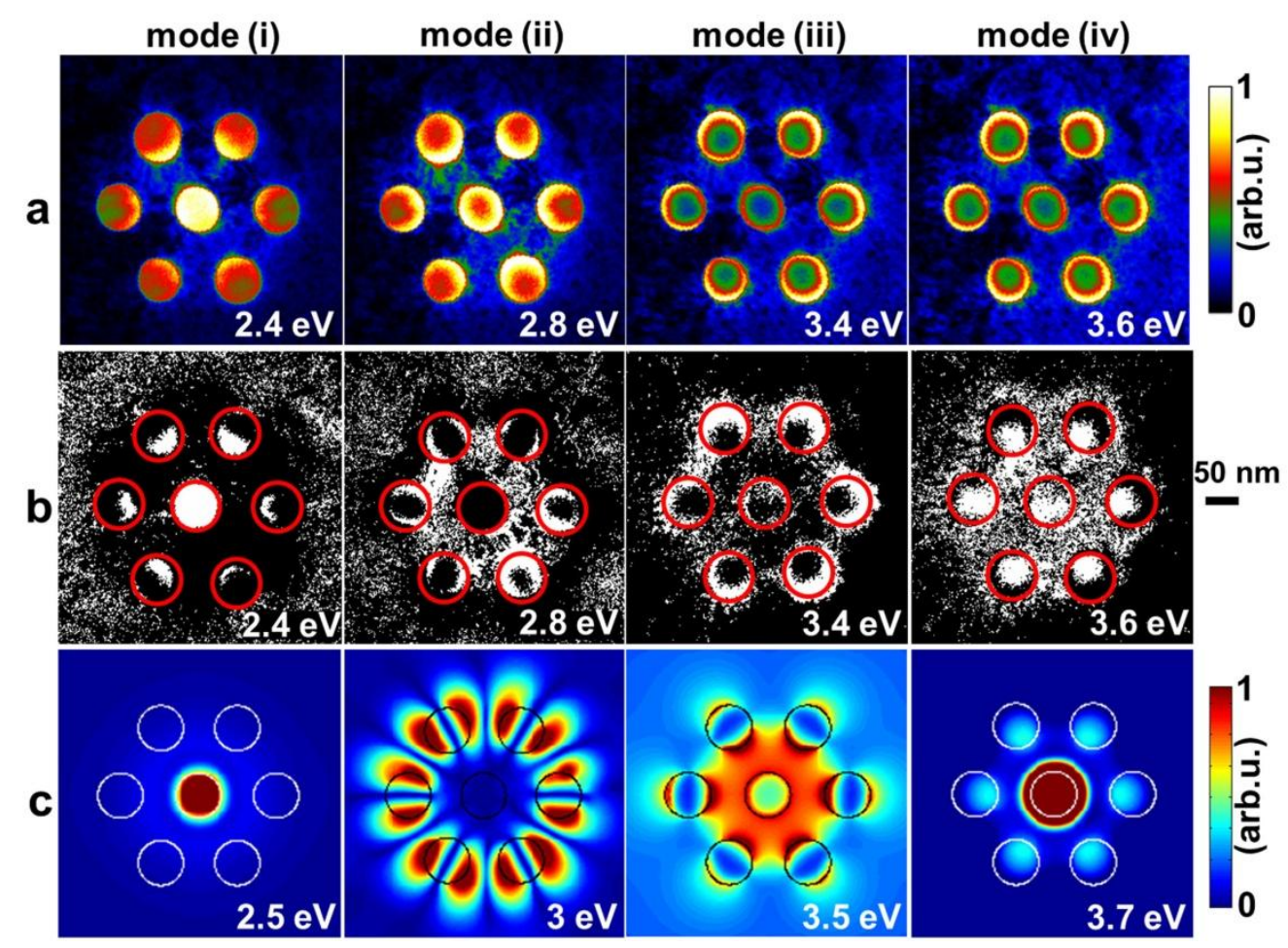

Figure 2. (a) Collection of modes acquired in EFTEM experiment at energy losses of (i) 2.4, (ii) 2.8, (iii) 3.4 , and (iv) $3.6 \mathrm{eV}$. The color code indicates the energy-loss probability in EFTEM images increasing from blue to yellow. (b) Peak maps obtained from the acquired images in (a). (c) Simulated modes with 3D-FDTD. Displayed is the modulus of the electric field $(|E z|)$, color coded increasing from blue to red, at (i) $2.5 \mathrm{eV}$, (ii) $3 \mathrm{eV}$, (iii) $3.5 \mathrm{eV}$, and (iv) $3.7 \mathrm{eV}$. The scale bar applies to all images. 